\title{
Relativistic effects in model calculations of double parton distribution functions
}

\author{
Matteo Rinaldi ${ }^{1}$ and Federico Alberto Ceccopieri ${ }^{2,3}$ \\ ${ }^{1}$ Instituto de Fisica Corpuscular (CSIC-Universitat de Valencia), Parc Cientific UV, \\ C/ Catedratico Jose Beltran 2, E-46980 Paterna (Valencia), Spain. \\ ${ }^{2}$ Dipartimento di Fisica e Geologia, Università degli Studi di Perugia and Istituto Nazionale di Fisica Nucleare, \\ Sezione di Perugia, via A. Pascoli, I - 06123 Perugia, Italy \\ ${ }^{3}$ IFPA, Universitè de Liège, B4000, Lìege, Belgium
}

(Dated: November 9, 2018)

\begin{abstract}
In this paper we consider double parton distribution functions (dPDFs) which are the main non perturbative ingredients appearing in the double parton scattering cross section formula in hadronic collisions. By using recent calculation of dPDFs by means of constituent quark models within the so called Light-Front approach, we investigate the role of relativistic effects on dPDFs. We find, in particular, that the so called Melosh operators, which allow to properly convert the LF spin into the canonical one and incorporate a proper treatment of boosts, produce sizeable effects on dPDFs. We discuss specific partonic correlations induced by these operators in transverse plane which are relevant to the proton structure and study under which conditions these results are stable against variations in the choice of the proton wave function.
\end{abstract}

\section{INTRODUCTION}

A proper description of final states in hadronic collisions requires the inclusion of multiple partonic interactions (MPI) [1 3] , i.e. a mechanism which takes into account the possibility that more than one couple of partons may interact in a given hadronic collisions. This possibility emerges naturally since both colliding hadrons are extended objects in transverse plane, at variance with processes involving point like probes, as in Deep Inelastic Scattering where, to date, no MPI effects have been reported. Multiple parton interactions enhance particle yields at low transverse momenta, affecting multiplicities and energy flows. MPI play an important role also in events characterized by an hard scale where they may contaminate the primary event with production of secondaries which contribute to the so called underlying event. In recent years, given the LHC operation, renewed interest has been paid to double parton scattering (DPS), in which a couple of partons from each hadron interacts between each other. If both interactions are hard enough, perturbative techniques can be applied and, as such, this class of processes need to be well controlled since they might represent a background to New Physics Searches. At the same time DPS has its own physical interest being sensitive to the nucleon structure. In particular, the cross section for this process depends on non-perturbative quantities, the so called double parton distribution functions (dPDFs). The latter encode the probability of finding two interacting partons, with longitudinal momentum fraction, w.r.t. the proton one, $x$, and relative transverse distance $\vec{b}_{\perp}$, offering the opportunity to investigate parton momentum and spin correlations in the nucleon, unveiling new information on the its structure, see Ref. [4]. Since dPDFs are two-body distributions, this knowledge is complementary to the one encoded in other type of (one-body) distributions, such as generalized parton distributions (GPDs) and transverse momentum dependent distributions (TMDs). To date, dPDFs are very poorly known objects. Little guidance on their structure come from sum rules which relate them to ordinary PDFs, see Refs. [5, 6], while their perturbative QCD evolution is still debated due to the presence of the so called inhomogeneous term in the evolution equations, see Refs. 7 [10]. In this situation it is clear that a proper theoretical modelization of DPS signal is quite challenging. This problem has been circumvented expressing the DPS cross section, $\sigma_{D P S}$, with final state $A+B$, by the following ratio, see e.g. Ref. [12]:

$$
\sigma_{D P S}^{A+B}=\frac{m}{2} \frac{\sigma_{S P S}^{A} \sigma_{S P S}^{B}}{\sigma_{e f f}}
$$

where $m$ is combinatorial factor depending on the final states $A$ and $B(m=1$ for $A=B$ or $m=2$ for $A \neq B)$ and $\sigma_{S P S}^{A(B)}$ is the single parton scattering cross section with final state $A(B)$. The ratio in Eq. (11) relies essentially on the assumption that the two hard scattering can be factorized, an hypothesis which has been investigated in detail for the double Drell-Yan process in Ref. [11]. Furthermore dPDFs are often built up in a full factorized form of the type:

$$
\begin{aligned}
F_{a b}\left(x_{1}, x_{2}, b_{\perp}, Q^{2}\right) & \sim f_{a}\left(x_{1}, Q^{2}\right) f_{b}\left(x_{2}, Q^{2}\right) \\
& \times\left(1-x_{1}-x_{2}\right)^{n} T\left(b_{\perp}\right),
\end{aligned}
$$

where $f_{a}\left(x_{1}, Q^{2}\right)$ and $f_{b}\left(x_{2}, Q^{2}\right)$ are the standard PDFs evaluated at the scale $Q^{2}, n \geq 0$ is a phenomenological parameter which takes into account possible phase space effects on the kinematic boundary, see Ref. 5], and the function $T\left(b_{\perp}\right)$ captures parton correlations in the tranverse plane. This ansatz for dPDFs exploits the idea that, for decreasing parton fractional momenta, $x$, the parton population in the nucleon grows up, resulting in 
a substantial longitudinal decorrelation of the joint distribution $F_{a b}$. The double parton interaction rate is then totally encapsulated in the function $T\left(b_{\perp}\right)$. In such a factorized approach, the effective cross section appearing in Eq. (11) is simply given by

$$
\sigma_{e f f}^{-1}=\int d^{2} b_{\perp}\left[T\left(b_{\perp}\right)\right]^{2}
$$

and, by construction, does not show any dependence on parton fractional momenta, hard scales or parton species. Due to the rather easy technical implementation of Eq. (1) and the almost total inclusiveness of the experimental analyses performed so far, all the present knowledge on DPS cross sections has been condensed in the experimental and model dependent extraction of $\sigma_{\text {eff }}$ [12 19]. To date, the corresponding number determined so far $\left(\sigma_{e f f} \simeq 15 \mathrm{mb}\right)$ is compatible, within errors, with a constant, irrespective of centre-of-mass energy of the hadronic collisions and final state $(\mathrm{A}+\mathrm{B})$ considered. Given this situation, many features of dPDFs are essentially unconstrained. It is therefore clear that non perturbative methods may give access to some relevant properties on these distributions 20 25], allowing, for example, to establish to which extent such dPDFs models may correctly reproduce the magnitudo of the transverse correlation encoded in $\sigma_{\text {eff }}$, see for instance results of Refs. [26, 27] on this point.

In this work, starting from the results obtained in Ref. [22], where dPDFs have been calculated in valence region within a fully relativistic covariant treatment, the so called Light-Front (LF) approach, we identify model independent effects induced on dPDFs by the relativistic treatment, in particular the violation of the factorized ansatz and the effects of parton correlation in the transverse plane in the proton structure. We also try to quantify the corresponding impact on observable-related quantities.

The paper is organized as follows. In Section [II we outline the structure of dPDFs and relativistic operators. In Section III we describe the details of the hadronic models used in the analysis. In Section IV we discuss the relevant issue of dPDFs factorization in longitudinal and transverse space and the impact of the correct treatment of relativistic effects on dPDFs. We finally draw our conclusions in Section $\mathrm{V}$.

\section{THE LIGHT-FRONT APPROACH AND RELATIVISTIC EFFECTS}

Following Ref. 22], dPDFs have been calculated starting from their Light-Cone correlator, which formally defines them in QCD. A suitable expression for dPDFs has been presented in Ref. [22]:

$$
\begin{aligned}
F\left(x_{1}, x_{2}, \vec{k}_{\perp}\right) & \propto \int d \vec{k}_{1} d \vec{k}_{2} \Psi\left(\vec{k}_{1}+\frac{\vec{k}_{\perp}}{2}, \vec{k}_{2}-\frac{\vec{k}_{\perp}}{2}\right) \\
& \times \Psi^{\dagger}\left(\vec{k}_{1}-\frac{\vec{k}_{\perp}}{2}, \vec{k}_{2}+\frac{\vec{k}_{\perp}}{2}\right) \\
& \times \delta\left(x_{1}-\frac{k_{1}^{+}}{M_{0}}\right) \delta\left(x_{1}-\frac{k_{1}^{+}}{M_{0}}\right) \\
& \times\left\langle S U(6)\left|D_{1}^{\dagger} D_{1} D_{2}^{\dagger} D_{2}\right| S U(6)\right\rangle,
\end{aligned}
$$

where $\vec{k}_{i}$ is the intrinsic three-momentum of the $i-$ parton, $k_{\perp}$ is the relative transverse momentum between the two active partons, $\Psi$ is the proton wave function in momentum space and $|S U(6)\rangle$ is the spin-flavor state evaluated according the commonly adopted $S U(6)$ symmetry. Here, as in Ref. 22], a factorization between the spin and the spatial part of the proton wave function is assumed. Let us remark that, thanks to this rigorous approach, the correct support of dPDFs is fulfilled, i.e. the dPDF vanish in unphysical regions, i.e. $x_{1}+x_{2}>1$. A proper inclusion of relativistic effects is obtained via the so called Light-Front (LF) approach. This is a common procedure, largely used for the calculation non perturbative distributions, see e.g. Refs. 28 33]. It should be noticed that $\mathrm{dPDFs}$, calculated in momentum space, describe a system where two partons have a relative transverse momentum $\left( \pm \vec{k}_{\perp}\right)$. This unbalance physically arises since the difference of parton transverse momenta is not conserved between the amplitude and its complex conjugate [34, 35]. Due to this unbalance, the dPDFs are not densities in this representation and they can not be interpreted as probabilistic distributions. In order to deal with distributions which admit a probabilistic interpretation we consider the Fourier transform of Eq. (4) w.r.t. to $\vec{k}_{\perp}$ which reads

$$
F\left(x_{1}, x_{2}, \vec{b}_{\perp}\right)=\int \frac{d \vec{k}_{\perp}}{(2 \pi)^{2}} e^{i \vec{k}_{\perp} \cdot \vec{b}_{\perp}} F\left(x_{1}, x_{2}, \vec{k}_{\perp}\right),
$$

with $\vec{b}_{\perp}$ being the relative transverse distance between the two partons. In this paper we only consider the distribution of two unpolarized quarks of flavor $u$ (c.f.r. Refs. $[21,22,[36])$ so that $F\left(x_{1}, x_{2}, \vec{k}_{\perp}\right) \equiv F_{u u}\left(x_{1}, x_{2}, k_{\perp}\right)$ depends only on $k_{\perp}=\left|\vec{k}_{\perp}\right|$. Due to rotational invariance of $F_{u u}$ in case of unpolarized quarks, the Fourier transform reduces to:

$$
F\left(x_{1}, x_{2}, b_{\perp}\right)=\frac{1}{2 \pi} \int d k_{\perp} k_{\perp} J_{0}\left(b_{\perp} k_{\perp}\right) F\left(x_{1}, x_{2}, k_{\perp}\right),
$$

being $J_{0}$ the Bessel function of the first kind and $b_{\perp}=$ $\left|\vec{b}_{\perp}\right|$. Anticipating some results discussed in the next Sections, we present in Fig. (1) the $b_{\perp}$-dependence of dPDFs at $x_{1}=0.2, x_{2}=0.3$. The plots show the probability for two partons to initiate two separate hard scattering as a function of their relative transverse distance, 

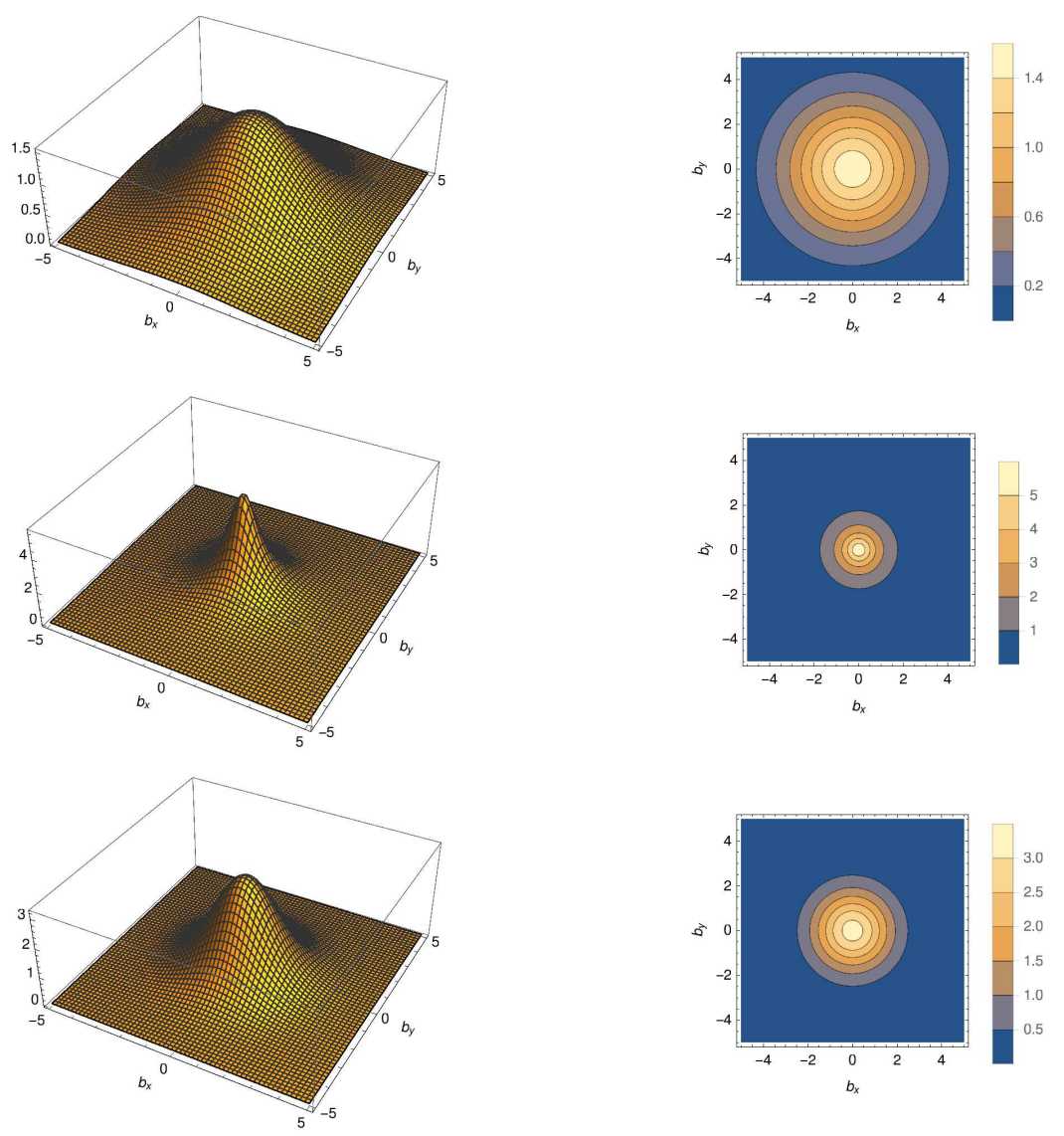

FIG. 1: Distribution evaluated via Eq. (6) by using different hadronic models presented in Section III in particular the NR (top), the $\mathrm{RL}$ (middle) and $\mathrm{HO}_{r e l}$ (bottom) models at $x_{1}=0.2, x_{2}=0.3$.

a unique information which is only accessible with such distributions. The same distribution for dPDFs with longitudinal and transversally polarized quarks are likely to show departure from this symmetric structure giving access to new details of the proton structure. These spin effects on dPDFs are presently under investigation and will reported in a separated paper. In Eq. (4), the canonical proton wave function is calculated by means of constituent quark models (CQM). The LF proton wave function, which naturally arises in the LF approach, see e.g. Ref. [28], is related to the canonical one thanks to the introduction of the Melosh rotations [37] which appear in the last line of Eq. (4). The latter quantities are related to LF boosts which, in such an approach, are kinematical operators. Formally they are defined as

$$
\hat{D}_{i}=\frac{m+x_{i} M_{0}+i\left(k_{i x} \sigma_{y}-k_{i y} \sigma_{x}\right)}{\sqrt{\left(m+x_{i} M_{0}\right)^{2}+k_{i x}^{2}+k_{i y}^{2}}},
$$

where $m$ is the constituent quark mass, $x_{i}$ the longitudinal momentum fraction carried by the $i$ quark, $\sigma_{x}$ and $\sigma_{y}$ are Pauli sigma matrices and $M_{0}$ is the energy that the proton had if quarks were free and it depends itself on $\vec{k}_{i \perp}$ and $x_{i}$. In particular, the Melosh operators are rotations between the rest frame of the system reached through the Light-Front boost or canonical boost and allow to rotate Light-Front spin into the canonical one. For example a Light-Front state with momentum $k$ and spin $\sigma,|k, \sigma\rangle_{L F}$ can be written in term of canonical one, $|k, \mu\rangle_{I F}$ as follows :

$$
|k, \sigma\rangle_{L F} \propto \sum_{\mu}\langle\mu|\hat{D}| \sigma\rangle|k, \mu\rangle_{I F}
$$

Thanks to this property, as pointed in Ref. [28], one can convert the Light-Front proton wave function into the canonical one. This procedure is suitable for the calculations of non perturbative quantities, such as parton distributions, since the proton wave function is usually evaluated by using the canonical Instant-Form approach.

From Eq. (77), it is clear that the structure of such operators induce non trivial correlations between the relevant variables at any energy scales. In order to visualize the effects produced by the term introduced in the last line of Eq. (4), one can analytically evaluate:

$$
\begin{aligned}
& D D^{\dagger}\left(\vec{k}_{\perp}, x_{1}, x_{2}, \vec{k}_{1 \perp}, \vec{k}_{2 \perp}\right)= \\
& \left\langle S U(6)\left|D_{1}^{\dagger} D_{1} D_{2}^{\dagger} D_{2}\right| S U(6)\right\rangle .
\end{aligned}
$$




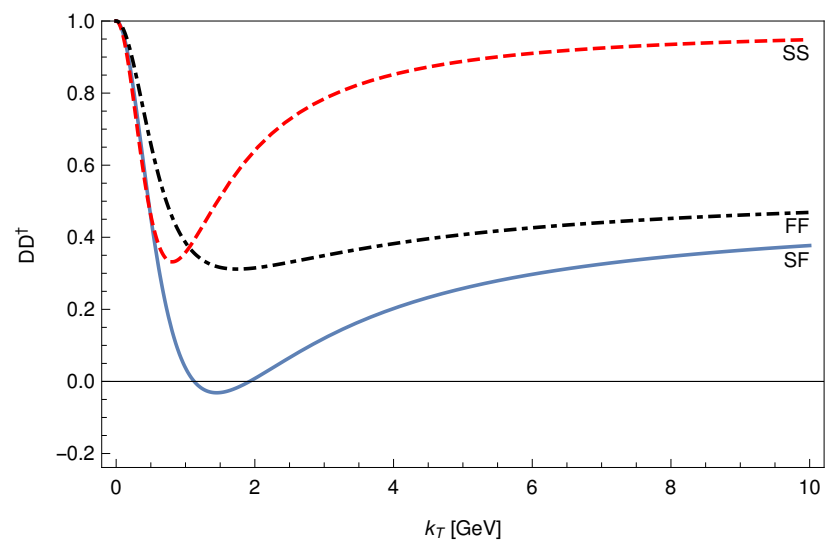

FIG. 2: The quantity Eq. (9), as function of $k_{T}=k_{\perp}$, evaluated in different regions of $x_{1}$ and $x_{2}$ with $\vec{k}_{1 \perp}=\vec{k}_{2 \perp}=0$.

However, since the dependence of the Melosh rotation w.r.t. all the variables expressed in Eq. (9) is quite complicated, one can, without loss of generality, examine its behavior in the limit $D D^{\dagger}\left(\vec{k}_{\perp}, x_{1}, x_{2}, \vec{k}_{1 \perp}=0, \vec{k}_{2 \perp}=0\right)$. For this calculation, the allowed phase space $x_{1}+x_{2} \leq 1$, here and in the following Sections, is sampled in three different couples of points, which we found representative for the effects we wish to discuss. In particular, we consider two fast partons (FF) with $x_{1}=0.2, x_{2}=0.3$, one slow and and one fast parton (SF) with $x_{1}=0.04, x_{2}=$ 0.3 and two slow partons (SS) with $x_{1}=0.04, x_{2}=0.03$. The calculation of Eq. (9) with these kinematic settings is presented in Fig. (2) where one may identify three distinct regions as a function of $k_{\perp}$. For $k_{\perp} \rightarrow 0$ the Melosh's in all kinematic configurations reduce to unity. In an intermediate region of $k_{\perp}$ the curves show a dip whose depth depends on the chosen kinematic configuration and, in particular, becoming negative in the SF configuration. At larger $k_{\perp}$ the curves flattens out with different asymptotics. This complicated pattern, generated by Melosh's rotations, affects the calculation of $\mathrm{dPDFs}$, which, in general, are distributions evaluated also at $k_{\perp} \neq 0$. It is worth noticing that such complicated behavior is due to the mixed combination of the four Melosh operators combined with the proton spin structure assumed and described in its wave function. These kind effects can not be observed in known quantities such as standard PDFs or, e.g., in momentum distributions, being all these distributions depending on diagonal matrix elements (i.e. evaluated at $k_{\perp}=0$ ). In this case, in fact, the product of two Melosh reduces to the unity. However, important effects due to the Melosh can be observed in model calculations of polarized PDFs. A crucial consequence of the presence of such operators is the difference between the longitudinal and transversely polarized PDFs. In fact, since boosts commute with rotations in the non relativistic limit, such distributions are identical in this framework, see details on e.g. [31]. Moreover, important effects are also found in the calculations of GPDs, see e.g. Refs. [28, 29, 31], where some distri-

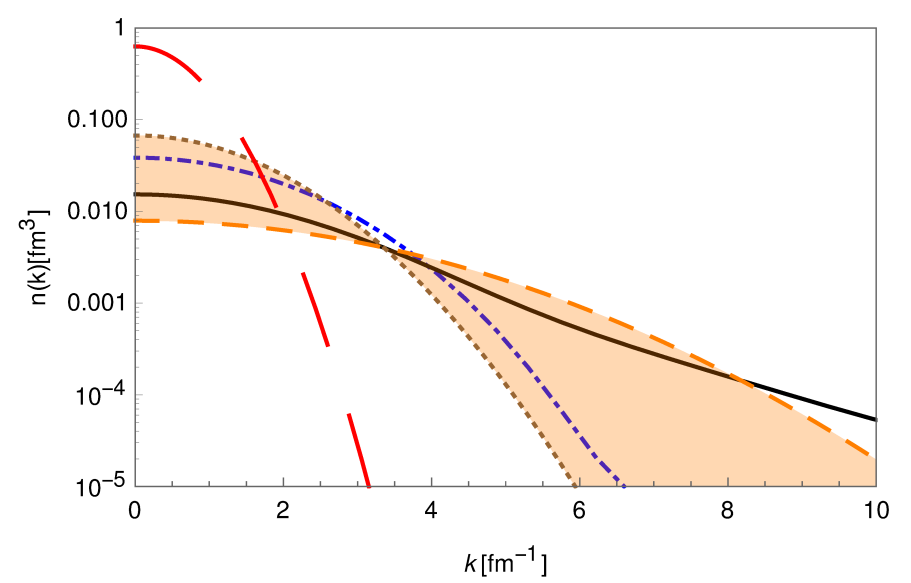

FIG. 3: Quark momentum distributions of the different models used in this analysis. RL (full black), NR (dot-dashed blue), Original $\mathrm{HO}$ (dashed red), modified $\mathrm{HO}_{\text {nrel }}$ (gold dotted), modified $\mathrm{HO}_{\text {rel }}$ (orange dashed). The orange band corresponds to variation of the parameter $6<\alpha^{2}<25 \mathrm{fm}^{-2}$.

butions are different from zero thanks to the presence of the Melosh. Such conclusions are also found in the analyses of TMDs in Light-Front CQM calculations Ref. [32]. Let us mention that Melosh effects can be appreciated in the calculation of nuclear spectral function and structure functions of the ${ }^{3} \mathrm{He}$ within the Light-Front approach, see Refs. [38, 39].

It should be noted, however, that the actual impact of Melosh on dPDFs is weighted by the chosen proton wave function and, in particular, by its structure at large parton momenta $k$. We address this issue in the next Section.

\section{HADRONIC MODELS}

The calculation of dPDFs via Eq. (44) involves, beside the relativistic boosts just described, the modeling of the (canonical) proton wave function which is obtained by means of CQM. The parameters of these models are fixed by comparison with subset of available data, e.g. , the hadronic spectrum or the proton electromagnetic form factor at small momentum transfer. Since the aim of the present analysis is to identify potential model independent effects on dPDFs, we consider a variety of proton wave functions. The first model is the so called Hypercentral quark model in both its relativistic (RL), Ref. [40], and non relativistic (NR), Ref. [41], versions. The quark momentum distributions obtained within these two models are shown in Fig. (3). The RL version (solid black) shows a broad tail extending at high momentum (hence relativistic) while the NR version (dot-dashed blue line) drops far more quickly at large momentum (hence non-relativistic). Since both versions assume a similar potential, we also consider a modified version of the harmonic oscillator (HO), see details on the proton wave function calculated in such model in 


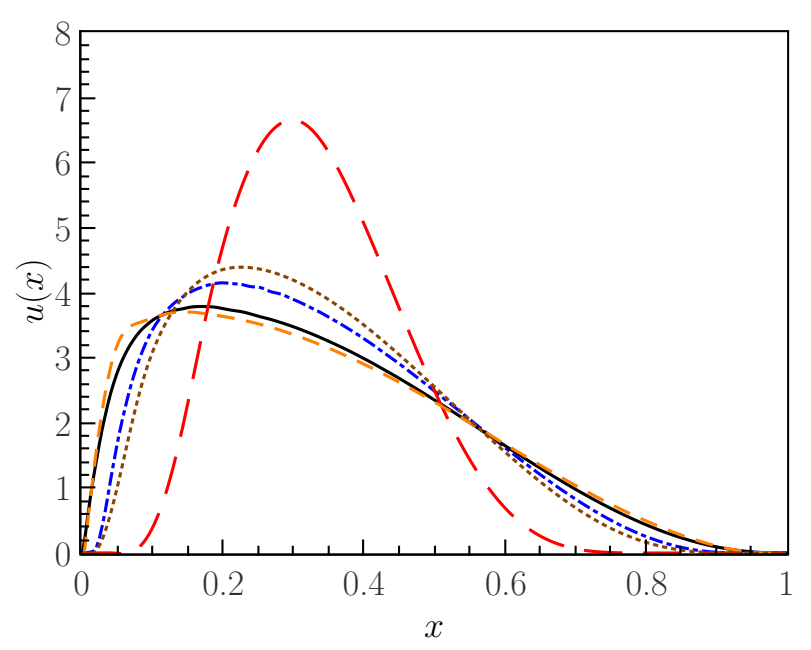

FIG. 4: Single parton distributions calculated with the different models used in this analysis. $R L$ full black line, NR dot-dashed blue line, Original $\mathrm{HO}$ dashed red line, modified $\mathrm{HO}_{\text {nrel }}$ gold dotted line, modified $\mathrm{HO}_{\text {rel }}$ orange dashed line.

Ref. 21]. In its original version, the width of the Gaussian structure of the proton wave function is fixed to $\alpha^{2}=1.35 \mathrm{fm}^{-2}$. As one can see in Figs. (3), the corresponding momentum distribution (red, long dashed line) decreases rather quickly w.r.t. $|\vec{k}|$, the quark momentum, showing a rather extreme non relativistic behavior, not suitable for the estimate of relativistic effects. Given the relative mathematical simplicity of such a model, we may construct a class of models of this type just varying the tunable parameter $\alpha$ in order to reproduce a momentum distribution which can have either a relativistic or non relativistic behavior. As shown in Fig. (3), we find that with the choice $\alpha^{2}=\alpha_{n r e l}^{2}=6 \mathrm{fm}^{-2}$, we can simulate a non relativistic model $\left(\mathrm{HO}_{\text {nrel }}\right)$, while with the choice $\alpha^{2}=25 \mathrm{fm}^{-2}$, the model $\left(\mathrm{HO}_{r e l}\right)$ develops a quite broad relativistic tail. Let us stress that for such values the agreement between HO model predictions and available experimental data is lost. We emphasize that the behavior of the CQM models, at large parton momentum, determines the behavior at small $x$ of the corresponding parton distributions functions. This feature is easily explained considering the definition of the longitudinal momentum fraction carried by a quark in the LF approach:

$$
x_{1}=\frac{k_{1}^{+}}{k_{1}^{+}+k_{2}^{+}+k_{3}^{+}}
$$

where the light cone notation has been introduced, $a^{+}=$ $a^{0}+a^{3}$ with $a^{\mu}$ being a generic four vector. Since in Eq. (10) it is always $k^{+}>0$, the extreme small $x$ region can be achieved only if one parton has a very high momentum. Therefore a fast drop of quark momentum distributions at large $|\vec{k}|$, that is a non relativistic behaviour, determines a smooth vanishing of PDFs as $x \rightarrow 0$. On the contrary, PDFs corresponding to relativistic models still

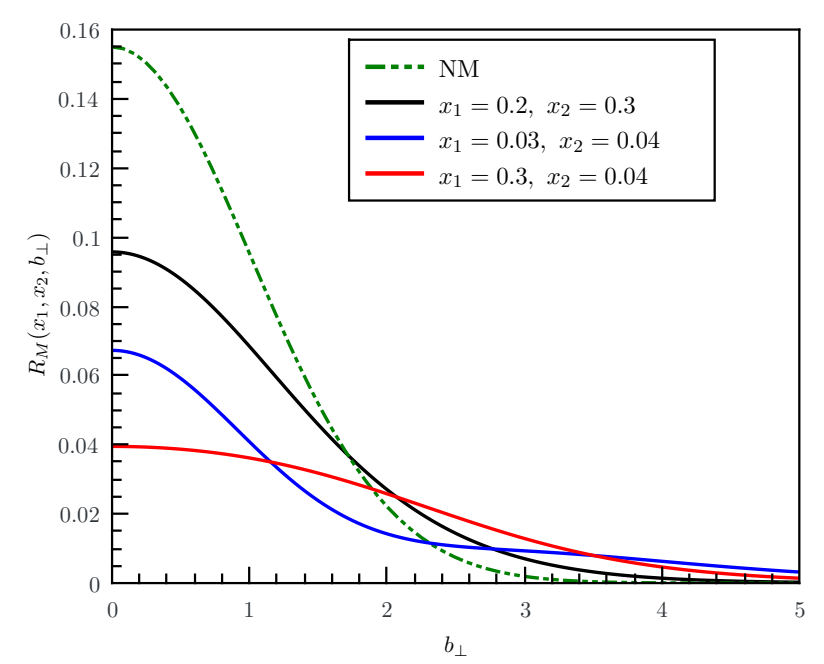

FIG. 5: The ratio (11) evaluated using the $\mathrm{HO}_{\text {rel }}$ model in three different regions of $x_{1}$ and $x_{2}$ as function of $b_{\perp}=\left|\vec{b}_{\perp}\right|$. In the legend the acronym "NM" specifies the calculation in which the Melosh rotations are neglected.

vanish in the limit $x \rightarrow 0$ but with a much harder behavior. All these results are summarized in Fig. (4) where the $u$-quark distributions, obtained from all the considered models, are compared together. With this selection of models, based on different potentials and showing different relativistic behavior, we now turn to the evaluation and discussion of dPDFs.

\section{CALCULATIONS OF DPDFS}

\section{A. Breaking the factorized ansatz}

As already mentioned in the previous sections, the present knowledge on the DPS cross section is quite inclusive, being based on the approximations in Eqs. (113), and it does not allow, at the moment, to pin down peculiar features of these distributions. Given this situation, the modeling of dPDFs aims to maximally exploit the current knowledge on the proton structure, assuming a fully factorized form in all the relevant variables, as indicated in Eq. (2). It is therefore clear that the modelization of dPDFs, beyond the approximation in Eq. (2), will require more differential DPS measurements. Such a factorized ansatz is nevertheless valuable since, under a number of approximations, it allows to relate the double gluon distribution functions to the gluon GPDs, exactly in the kinematic range of small $x$ where CQM model are difficult to extend and the corresponding theoretical predictions for $T\left(b_{\perp}\right)$ are lacking, see Refs. [34, 35]. On the perturbative side, the evolution effects on such a factorized $b$-space ansatz have been investigated in Ref. [42]. In the moderate large values of fractional momenta, $x$, natural domain of CQM with realistic potentials, as dis- 

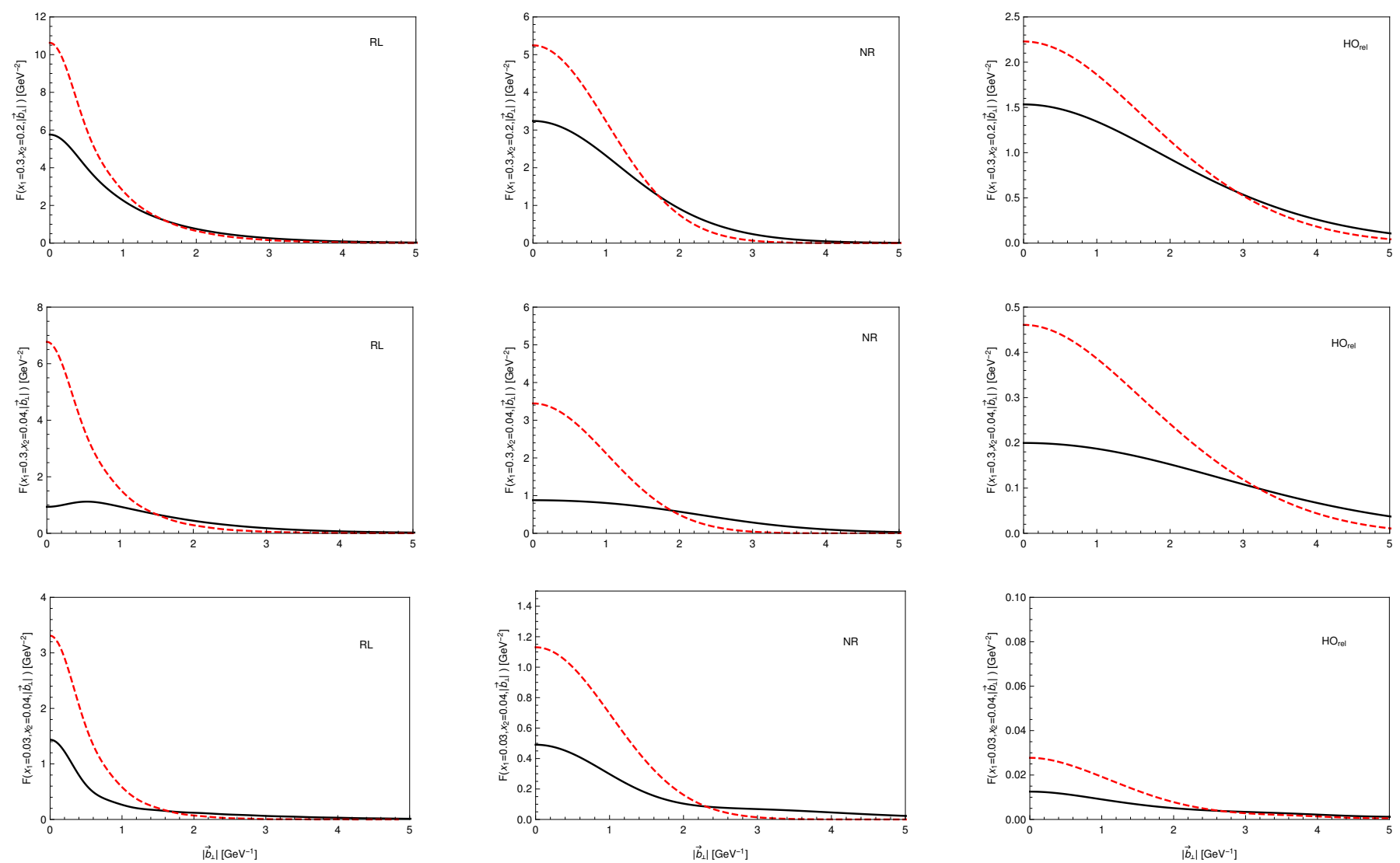

FIG. 6: The distribution Eq. (6) as a function of $\left|\vec{b}_{\perp}\right|$ evaluated in three different kinematical configurations (from top to bottom $F F, S F, S S$, respectively) using the relativistic model, the $\mathrm{NR}$ one and the $\mathrm{HO}_{\text {rel }}$ one (from left to right, respectively). Lines correspond to the evaluation of Eq. (6) with (black) and without (red) Melosh's.

cussed in Refs. [21, 22, 36], calculations show that both the factorization of dPDFs as a product of single parton distributions and, perhaps more interestingly, the $\left(x_{1}, x_{2}\right)-k_{\perp}$ factorization are violated. For recent results on the breaking of the factorization on the $x_{1}$ and $x_{2}$ dependence, see Ref. [43]. Furthermore the breaking of the $\left(x_{1}, x_{2}\right)-k_{\perp}$ factorization might be generated both by the specific form of the proton wave function and by relativistic effects induced by Melosh operators. Within this context, the harmonic oscillator model appears to be particularly suitable to quantify to which extent such factorization breaking are due to relativistic effects alone. Within this model, in fact, the $\left(x_{1}, x_{2}\right)-k_{\perp}$ dependences are entirely factorized, see Ref. 21]. In order to estimate these effects quantitatively, we evaluate the ratio

$$
R_{N}\left(x_{1}, x_{2}, b_{\perp}\right)=\frac{F\left(x_{1}, x_{2}, b_{\perp}\right)}{\int d \vec{b}_{\perp} F\left(x_{1}, x_{2}, b_{\perp}\right)} .
$$

It is worth to notice that, according to Eq. (5), $\int d \vec{b}_{\perp} F\left(x_{1}, x_{2}, b_{\perp}\right)=F\left(x_{1}, x_{2}, k_{\perp}=0\right)$ so that the denominator in Eq. (11) does not depend on the Melosh rotations which reduce to unity in the $k_{\perp} \rightarrow 0$ limit, see Fig. (2). The ratio, Eq. (11), calculated by using the $\mathrm{HO}_{r e l}$, is presented in Fig. (5). The ratio $R_{N}$ with dPDFs evaluated without the Melosh rotations gives identical (superimposed) results in the three kinematic regions of $x_{1}$ and $x_{2}$, as expected. On the contrary, if Melosh rotation are taken into account, we observe a significant reduction of the magnitudo of $\mathrm{dDPFs}$ and a progressive broadening of the $b_{\perp}$-dependence w.r.t. the distribution without Melosh depending on the partonal fractional momenta $x_{i}$. This effect is sizeable especially in the SF configuration. This observation leads us to conclude that for dPDFs evaluated through models (which themselves may or may not show a $\left(x_{1}, x_{2}\right)-k_{\perp}$ factorization) via Eq. (44), at the hadronic scale, relativistic effects induce significant factorization breaking effects. In light of this result, it is worth to remark that possible modulation in the $b_{\perp}$-space, depending on parton fractional momenta, might not be disregarded altogether.

\section{B. Relativistic effects}

It appears from the last Section that Melosh rotations do not allow, in general, to factorize dPDFs in a longitudinal and transverse distributions. More importantly they cause a significant reduction of the distributions, 

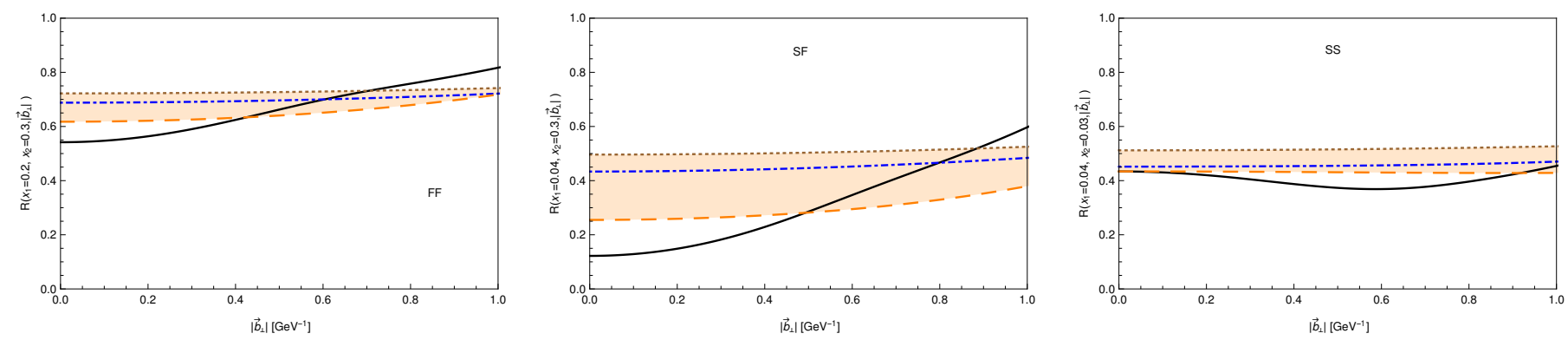

FIG. 7: The ratio Eq. (12) evaluated as function of $\left|b_{\perp}\right|$ in different kinematic regions: $x_{1}=0.2, x_{2}=0.3$ (left panel) and $x_{1}=$ $0.04, x_{2}=0.3$ (middle panel) and $x_{1}=0.04, x_{2}=0.03$ (right panel). RL full black line, NR dot-dashed blue line, Original HO dashed red line, modified $\mathrm{HO}_{n r e l}$ gold dotted line, modified $\mathrm{HO}_{\text {rel }}$ orange dashed line. The orange band corresponds to variation of the parameter $6<\alpha^{2}<25 \mathrm{fm}^{-2}$.

which, in turn, induce substantial variation of the corresponding DPS cross section. In order to further investigate these effects, in this section we calculate dPDFs in $b_{\perp}$-space via Eq. (6) with and without Melosh rotations. We note that in the latter case we basically reduce to the results presented in Refs. [20 22]. The results of these calculations are shown in Fig. (6), where predictions from different models are presented in columns and different kinematical configurations in rows. The $b_{\perp}$ spectra without Melosh rotation (NM) show a great variety in magnitudo and width, reflecting the difference in the used proton wave functions. In all cases the distributions are peaked at $b_{\perp}=0$ and show a finite behaviour in the short distance limit. If Melosh rotations are included (red dotted lines), we observe a significant reduction of the magnitudo of the distributions. In the particular, in the SF kinematics, the magnitudo of the suppression is more pronunciated, and, for the RL models (left panel, middle row), the distribution tends to decrease as $b_{\perp} \rightarrow 0$ and it does show a maximum shifted to a non-vanishing value of $b_{\perp}$. We conclude that for relativistic models, in the $\mathrm{SF}$ region, these operators discourage the partons to be closed to each other. This model dependent behavior results from the combined effect of the negative contributions of the Melosh rotations in Eq. (6) and the large $|\vec{k}|$ tail of the RL model. The amount of the suppression, induced by the Melosh operators, is again conveniently quantified studying the ratio

$$
R\left(x_{1}, x_{2}, \vec{b}_{\perp}\right)=\frac{F\left(x_{1}, x_{2}, b_{\perp}\right)}{F_{N M}\left(x_{1}, x_{2}, b_{\perp}\right)},
$$

where here $F_{N M}\left(x_{1}, x_{2}, b_{\perp}\right)$ is the distribution in Eq. (4) once the Melosh rotations are neglected. The corresponding results are reported in Fig. (77), where it is shown that the suppression slightly depends on the kinematical configurations, being smaller in the FF one and, on average, around 0.5 in the FS and SS regions. Moreover such suppression is rather model independent, as can be inferred by the relatively contained spread of the orange band. In all the previous sections, the main effects of the Melosh rotations have been analyzed directly on the dPDFs, either in momentum or coordinate space. It is worth to remark, however, that dPDFs appear in the DPS cross section in a convolution like formula which reads [1],

$$
\sigma_{D P S}^{A+B} \propto \sum_{a b c d} \int d \vec{b}_{\perp} F_{a c}\left(b_{\perp}\right) F_{c d}\left(b_{\perp}\right) \hat{\sigma}_{a b}^{A} \hat{\sigma}_{c d}^{B}
$$

where we have neglected the dependences on longitudinal fractional momenta and $\hat{\sigma}$ are the elementary partonic cross sections for the process $a b(c d) \rightarrow A(B) X$. It is therefore clear that the details of the $b_{\perp}$ dependence of the dPDFs gets obscured by the convolution and it is intertwined with the dependences on longitudinal fractional momenta. Within this respect, we notice that a more direct access to the transverse structure of dPDFs may be provided analyzing the DPS component in multijet photoproduction in $e p$ or $p p$ collisions. In this case the quasi-real photon, emitted either by the electron or the proton and fluctuating in $q \bar{q}$ dipoles, probes parton pair in the nucleon at a relative transverse distance of the order $b_{\perp} \sim 1 / Q$ [44 46] while its low virtuality $Q$ can be controlled experimentally. In the present context, a proper calculation of $\sigma_{\text {eff }}$ via eq. (13) requires the selection of a definite final state $A+B$, the evaluation of corresponding partonic cross sections and the perturbative evolution of dPDFs from the hadronic scale (in the present work $\mu_{0}=0.1 \mathrm{GeV}^{2}$, where only three valence quarks carry the proton momentum) to scales $\mu_{A}$ and $\mu_{B}$ characterising the hard processes. We will report on these results in a separate publication. In the present work, in order to get a quantitative estimate of the possible role of the Melosh on observable-related quantities, following the lines of Refs. 26, 27], we define the ratio

$$
R_{\sigma}\left(x_{1}, x_{2}\right)=\frac{\int d \vec{b}_{\perp} F_{N M}\left(x_{1}, x_{2}, b_{\perp}\right)^{2}}{\int d \vec{b}_{\perp} F\left(x_{1}, x_{2}, b_{\perp}\right)^{2}},
$$

where the square is taken to mimic the analytic structure of the DPS cross section in Eq. (13) and Eq. (3). The ratio in Eq. (14) has been calculated by using the addressed models in the three kinematics configurations. The results are presented in in Tab. (II). One should notice that in regions where the three CQM are completely 


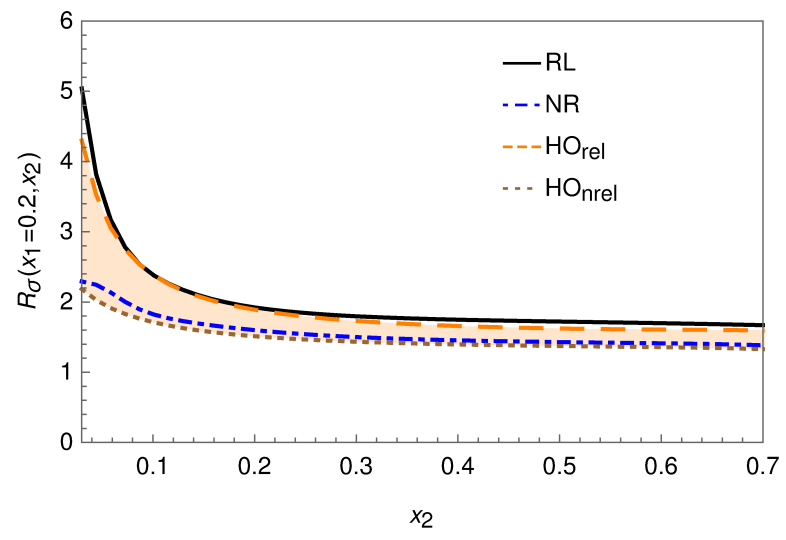

FIG. 8: The ratio $R_{\sigma}\left(x_{1}, x_{2}\right)$ calculated for fixed values $x_{1}$ as a function of $x_{2}$ within all the adopted models.

\begin{tabular}{|l|l|l|l|l|}
\hline & $\mathrm{RL}$ & $\mathrm{NR}$ & $\mathrm{HO}_{\text {rel }}$ & $\mathrm{HO}_{\text {nrel }}$ \\
\hline$R_{\sigma}\left(x_{1}=0.03, x_{2}=0.04\right)$ & 4.83 & 2.80 & 4.12 & 2.36 \\
\hline$R_{\sigma}\left(x_{1}=0.04, x_{2}=0.3\right)$ & 4.33 & 2.27 & 3.66 & 2.05 \\
\hline$R_{\sigma}\left(x_{1}=0.2, x_{2}=0.3\right)$ & 1.85 & 1.50 & 1.73 & 1.73 \\
\hline
\end{tabular}

TABLE I: The ratio $R_{\sigma}\left(x_{1}, x_{2}\right)$ calculated for different kinematical configurations and adopted models.

different, the effects of the Melosh are rather independent on the choice of the detailed proton structure considered. For the seek of transparency, some differences are found when small $x$ are involved in the calculation. This feature can be seen as a limit of the present analysis. In fact, as already mentioned, the low $x$ region is associated to high momenta, where the three CQM substantially differ from each other and details of the models can not be totally separated by those of the relativistic treatment. As shown in Fig. (8), the spread of $R_{\sigma}$ calculated within different models increases for decreasing $x_{2}$. This reflects different modelizations of the proton wave function at high quark momenta, see Fig. (4). Nevertheless, it should be noticed that the value of the ratio and its spread, induced by different models, becomes constant for, approximately, $x_{2}>0.1$. Therefore we may conclude that, in the valence region, the suppression factor (a factor around 2) induced by Melosh rotations is quite a model independent effect.

\section{CONCLUSIONS}

In conclusion, in this work a quantitative analysis of relativistic effects on $\mathrm{dPDF}$ s has been provided thanks to the correct treatment of dPDF in a relativistic framework due to the LF approach, which implies the introduction of the so called Melosh rotations in order to achieve a full Poincarè covariant description of dPDFs. We have discussed to which extent the Melosh rotations alone may affect the often assumed factorization in $\left(x_{1}, x_{2}\right)-k_{\perp}$ space, which is commonly used in the experimental analyses to extract the DPS cross section. We found that for relativistc models in the very low $x$ region, associated to high momenta of the quark, their effect is maximal and, for large unbalance of longitudinal momenta, they prevent the two interacting partons to be close to each other in transverse space. Then we have emphasized, by employing appropriate ratios, the role of such operators, and the degree of model independency of these results. In particular, such rotations produce a strong reduction of the size of the dPDFs, w.r.t. the same calculation where such operators are neglected. As a consequnce they affect quantities related to $\sigma_{\text {eff }}$ by a suppression factor which is, depending on the models, a factor ranging from 2 , in the FF configuration, to 4 in the SS one. In closing, we have found that relativistic effects on dPDFs are sizeable and they should be always taken into account in these kind of model calculations.

\section{Acknowledgements}

This work is supported in part through the project "Hadron Physics at the LHC: looking for signatures of multiple parton interactions and quark gluon plasma formation (Gossip project)", funded by the "Fondo ricerca di base di Ateneo" of the Perugia University. This work was supported in part by the Mineco under contract FPA2013-47443-C2-1-P and SEV-2014-0398.

We warmly thank Sergio Scopetta, Vicente Vento, Marco Traini, Jonathan Gaunt and Alberto Accardi for many useful discussions.
[1] N. Paver and D. Treleani, Nuovo Cim. A70, (1982) 215.

[2] M. Mekhfi, Phys. Rev. D32, (1985) 2371.

[3] T. Sjostrand and M. Van Zijl, Nuovo Cim. A 70, 215 (1982).

[4] G. Calucci and D. Treleani, Phys. Rev. D 60, 054023 (1999).

[5] J. R. Gaunt and W. J. Stirling, JHEP 03, 005 (2010).

[6] F. A. Ceccopieri, Phys. Lett. B 734 (2014) 79

[7] M. Diehl and A. Schafer, Phys. Lett. B 698 (2011) 389

[8] M. Diehl, D. Ostermeier and A. Schafer JHEP 03, 089
(2012).

[9] A. M. Snigirev, Phys. Rev. D 68 (2003) 114012

[10] F. A. Ceccopieri, Phys. Lett. B 697 (2011) 482

[11] M. Diehl et al., JHEP 01 (2016) 076.

[12] H. Jung \& al., Proceedings, 7th International Workshop on Multiple Partonic Interactions at the LHC (MPI@LHC 2015), https://bibpubdb1.desy.de/record/297386, DESY-PROC-2016-01

[13] T. Akesson et al. [Axial Field Spectrometer Collaboration], Z. Phys. C 34 (1987) 163. 
[14] J. Alitti et al. [UA2 Collaboration], Phys. Lett. B268, 145 (1991).

[15] F. Abe et al. [CDF Collaboration], Phys. Rev. D 56, 3811 (1997).

[16] V. M. Abazov et al. [D0 Collaboration], Phys. Rev. D 81, 052012 (2010).

[17] G. Aad et al. [ATLAS Collaboration], New J. Phys. 15, 033038 (2013).

[18] S. Chatrchyan et al. [CMS Collaboration], JHEP 03, 032 (2014).

[19] R. Aaij et al. [LHCb Collaboration], JHEP 1404 (2014) 091

[20] H. M. Chang, A. V. Manohar and W. J. Waalewijn, Phys. Rev. D 87, no. 3, 034009 (2013).

[21] M. Rinaldi, S. Scopetta and V. Vento, Phys. Rev. D 87, 114021 (2013).

[22] M. Rinaldi, S. Scopetta, M. Traini and V. Vento, JHEP 12, 028 (2014).

[23] W. Broniowski and E. Ruiz Arriola, Few Body Syst. 55, 381 (2014).

[24] W. Broniowski, E. Ruiz Arriola and K. Golec-Biernat, Few Body Syst. 57, no. 6, 405 (2016).

[25] T. Kasemets and A. Mukherjee, Phys. Rev. D 94 (2016) no.7, 074029

[26] M. Rinaldi, S. Scopetta, M. Traini and V. Vento, Phys. Lett. B 752, 40 (2016).

[27] M. Traini, S. Scopetta, M. Rinaldi and V. Vento, arXiv:1609.07242 [hep-ph].

[28] S. Boffi, B. Pasquini and M. Traini, Nucl. Phys. B 649, 243 (2003);

[29] S. Boffi, B. Pasquini and M. Traini, Nucl. Phys. B 680, 147 (2004);
[30] B. Pasquini, M. Traini and S. Boffi, Phys. Rev. D 71, 034022 (2005).

[31] B. Pasquini, M. Pincetti and S. Boffi, Phys. Rev. D 72, 094029 (2005)

[32] B. Pasquini, S. Cazzaniga and S. Boffi, Phys. Rev. D 78, 034025 (2008)

[33] M. Traini, Phys. Rev. D 89, no. 3, 034021 (2014).

[34] B. Blok, Y. Dokshitser, L. Frankfurt and M. Strikman, Eur. Phys. J. C 72, 1963 (2012).

[35] B. Blok, Y. Dokshitzer, L. Frankfurt and M. Strikman, Eur. Phys. J. C 74, 2926 (2014).

[36] A. V. Manohar and W. J. Waalewijn, Phys. Rev. D 85, 114009 (2012).

[37] H. J. Melosh, Phys. Rev. D 9, 1095 (1974).

[38] A. Del Dotto, E. Pace, G. Salmè and S. Scopetta, arXiv:1609.03804 [nucl-th].

[39] E. Pace, G. Salme, S. Scopetta, A. Del Dotto and M. Rinaldi, Few Body Syst. 54, 1079 (2013).

[40] P. Faccioli, M. Traini and V. Vento, Nucl. Phys. A 656 (1999) 400 .

[41] M. Ferraris, M. M. Giannini, M. Pizzo, E. Santopinto and L. Tiator, Phys. Lett. B 364, 231 (1995).

[42] M. Diehl, T. Kasemets and S. Keane, JHEP 05, 118 (2014).

[43] M. Rinaldi, S. Scopetta, M. C. Traini and V. Vento, JHEP 10, 063 (2016).

[44] B. Blok and M. Strikman, Eur. Phys. J. C74 (2014) no. 12,3214

[45] J. M. Butterworth, J. R. Forshaw and M. H. Seymour, Z. Phys. C72 (1996) 637

[46] J. R. Gaunt, JHEP 01 (2013) 042 